\title{
Rescue of retinal ganglion cells in optic nerve injury using cell-selective AAV mediated delivery of SIRT1
}

\author{
Ahmara G. Ross ${ }^{1,2} \cdot$ Devin S. McDougald ${ }^{2} \cdot$ Reas S. Khan ${ }^{1,2} \cdot$ Thu T. Duong ${ }^{1,2} \cdot$ Kimberly E. Dine $\mathbb{D}^{1} \cdot$ \\ Puya Aravand $\mathbb{C}^{1,2} \cdot$ Jean Bennett $\mathbb{C}^{1,2} \cdot$ Venkata Ramana Murthy Chavali ${ }^{1} \cdot$ Kenneth S. Shindler $^{1,2}$
}

Received: 5 March 2020 / Revised: 7 December 2020 / Accepted: 15 January 2021 / Published online: 15 February 2021

(c) The Author(s) 2021. This article is published with open access

\begin{abstract}
SIRT1 prevents retinal ganglion cell (RGC) loss in models of optic neuropathy following pharmacologic activation or genetic overexpression. The exact mechanism of loss is not known, prior evidence suggests this is through oxidative stress to either neighboring cells or RGC specifically. We investigated the neuroprotective potential of RGC-selective SIRT1 gene therapy in the optic nerve crush (ONC) model. We hypothesized that AAV-mediated overexpression of SIRT1 in RGCs reduces RGC loss, thereby preserving visual function. Cohorts of C57B1/6J mice received intravitreal injection of experimental or control AAVs using either a ganglion cell promoter or a constitutive promoter and ONC was performed. Visual function was examined by optokinetic response (OKR) for 7 days following ONC. Retina and optic nerves were harvested to investigate RGC survival by immunolabeling. The AAV7m8-SNCG.SIRT1 vector showed 44\% transduction efficiency for RGCs compared with $25 \%(P>0.05)$ by AAV2-CAG.SIRT1, and AAV7m8-SNCG.SIRT1 drives expression selectively in RGCs in vivo. Animals modeling ONC demonstrated reduced visual acuity compared to controls. Intravitreal delivery of AAV7m8-SNCG.SIRT1 mediated significant preservation of the OKR and RGC survival compared to AAV7m8-SNCG.eGFP controls, an effect not seen with the AAV2 vector. RGC-selective expression of SIRT1 offers a targeted therapy for an animal model with significant ganglion cell loss. Over-expression of SIRT1 through AAV-mediated gene transduction suggests a RGC selective component of neuro-protection using the ONC model. This study expands our understanding of SIRT1 mediated neuroprotection in the context of compressive or traumatic optic neuropathy, making it a strong therapeutic candidate for testing in all optic neuropathies.
\end{abstract}

\section{Introduction}

The irreversible effects of traumatic optic neuropathy (TON) can result from direct impulse or indirectly by shearing or stretch of the optic nerve [1]. Although this type of injury can occur in any age group, most TON occurs in

Supplementary information The online version contains supplementary material available at https://doi.org/10.1038/s41434021-00219-z.

Ahmara G. Ross

ahmara.ross@pennmedicine.upenn.edu

1 University of Pennsylvania/Ophthalmology, Philadelphia, PA, USA

2 Center for Advanced Retinal and Ocular Therapeutics, F. M. Kirby Center for Molecular Ophthalmology, Perelman School of Medicine, University of Pennsylvania, Philadelphia, PA, USA young males under the age of 18 , resulting in a range of visual outcomes from unaffected to substantial vision loss. The damage occurs at the level of axons, disrupting critical connections needed to maintain retinal ganglion cell (RGC) function. Mechanisms such as vascular and inflammatory mediators of RGC death have been implicated in perpetuating the process of RGC death, however the true pathophysiology is likely direct cell death [2]. Regardless, it is clear there is an unmet need for therapy aimed at delaying and potentially aborting the cell death associated with loss of vision.

Any primary insult to the visual tract or secondary deficits due to inflammation, demyelination, compression, or structural effects of the surrounding tissue can lead to RGC death and visual decline. Optic nerve crush (ONC) induces axonal degeneration, followed by a gradual death of RGCs, which results in irreversible vision loss [3], providing a fast, reproducible technique to observe RGC decline, as well as observe effects of novel neuroprotective therapeutic approaches [4]. 
A multitude of data has implicated a role for SIRT1induced mitochondrial biogenesis in RGC survival in ONC, inflammatory, and viral-induced optic neuropathies [4-7]. Enhanced activity and overexpression of this gene have shown delayed RGC death resulting in visual decline in multiple optic nerve injuries. Recently, increased expression of SIRT1 using AAV vectors with non-selective expression in retinal cells demonstrated a sustained and upward trend in neuro-protective capabilities [8] making this an excellent initial target gene for validating the use of this technology. This therapeutic intervention was not RGC specific.

The use of gene therapy as a delivery system to treat ophthalmic diseases continues to show promise as a powerful technique to introduce targeted changes into a cell with molecular surgical precision [9]. Ophthalmology represents an area where this technique has flourished due to the eye's accessible location and the ability of progress to be monitored in vivo with minimal damage to tissues. One particular area of ophthalmology that would benefit from gene targeting is optic neuropathy [10]. The mitochondrial biogenesis and RGC survival induced by SIRT1 in optic neuropathies makes it an excellent target gene for developing a gene therapy treatment.

In the present study, we compared the effects of SIRT1 overexpression in experimental ONC via AAV gene transfer to RGCs. We developed and characterized AAV7m8 vectors that drive RGC-specific expression of human SIRT1 in vitro and in the mouse retina. We examined the neuroprotective contribution of SIRTI gene augmentation in suppressing RGC death and vision loss in mice that have undergone ONC.

\section{Methods}

\section{Animals}

C57Bl/6J mice were obtained from the Jackson Laboratory and raised in a 12-h light/dark cycle. Animals were housed at the University of Pennsylvania in compliance with ARVO Statement for the Use of Animals in Ophthalmic and Vision Research as well as with institutional and federal regulations.

\section{Cell culture}

The human iPSCs were generated from keratinocytes or blood cells via polycistronic lentiviral transduction (Human STEMCCA Cre-Excisable constitutive polycistronic [OKS/ Myc] Lentivirus Reprogramming Kit, Millipore) by University of Pennsylvania iPSC Core facility and characterized with a hES/iPS cell pluripotency RT-PCR kit [11]. The induced pluripotent stem cell-derived retinal ganglion cells
(iPSC-RGCs) were derived using a modified protocol involving small molecules to inhibit BMP, TGF-B (SMAD) and Wnt signaling to differentiate RGCs from iPSCs [12]. The iPSC-RGCs cells with structural and functional features characteristic of native RGCs are used in our study.

We seeded iPSC-RGCs cells at a density of 350,000 cells and transduced with AAV2 vectors at a multiplicity of infection (MOI) of 100,000 vector genomes (vg) per cell. iPSC-RGCs were harvested $48 \mathrm{~h}$ post-transduction for immunocytochemistry analysis. Cells were rinsed with $1 \mathrm{X}$ PBS and fixed in $4 \%$ paraformaldehyde (PFA) for $15 \mathrm{~min}$ at room temperature. Afterwards, cells were blocked in $0.1 \%$ Triton X-100 and 1\% bovine serum albumin (BSA) for $30 \mathrm{~min}$ at room temperature. Cells were incubated with primary antibody solution in $1 \%$ BSA and rabbit anti-FLAG antibody (CST \#14793; 1:200), SIRT1 antibody (sc-74465; 1:200), or BRN3A (EP1972Y, 1:2000) for $1 \mathrm{~h}$ at room temperature. Cells were washed with $1 \mathrm{X}$ PBS and incubated in secondary antibody solution containing 1\% BSA and goat anti-rabbit AlexaFluor-594 antibodies (1:500) for $1 \mathrm{~h}$ at room temperature. Cells were removed from secondary incubation, washed in $1 \mathrm{X}$ PBS, and mounted with (Fluoromount-G; Southern Biotech; Birmingham, AL, USA) containing DAPI.

\section{AAV vector design and production}

Human SIRT1 (transcript variant 1) cDNA clones were obtained from Origene. Sequences were amplified with Q5 DNA polymerase (NEB) and cloned into an AAV expression plasmid using a commercial cloning kit (In-Fusion HD; Clontech Laboratories, Mountain View, CA, USA). Transgene expression was driven by either the CAG promoter derived from pDRIVE-CAG (InvivoGen, San Diego, CA, USA) [13] or the codon optimized SNCG (gamma-synuclein promoter) [13]. Both cDNA sequences contained a Cterminal 3xFLAG epitope tag that terminates into a bovine growth hormone polyadenylation sequence. AAV expression cassettes were flanked by the AAV2 inverted terminal repeats. A pro-viral plasmid driving expression of enhanced green fluorescent protein (eGFP) was used [8] and contains identical cis regulatory elements. AAV2-CAG.SIRT1, AAV2-CAG.eGFP, AAV7.m8-SNCG.SIRT1, AAV7m8SNCG.eGFP vectors were generated using previously described methods and purified with $\mathrm{CsCl}$ gradient by the CAROT research vector core at the University of Pennsylvania [14]. The AAV7m8 capsid plasmid was a kind gift from Dr. John Flannery (UC-Berkeley).

\section{Intravitreal injections}

4-week-old mice were anesthetized by isoflurane inhalation. A $33 \frac{1}{2}$ gauge needle was used to create a small incision at the 
limbus. Afterward, a 10-uL Hamilton syringe (701 RN; Hamilton Company, Reno, NV, USA) attached to a 33-gauge blunt-end needle was inserted into the vitreous cavity with the needle tip placed directly above the optic nerve head. We dispensed $2 \mathrm{uL}$ of AAV preparation containing approximately $1 \times 10^{10}$ vector genomes into each eye bilaterally. Vehicle treated eyes were injected with an equivalent volume of vector dilution buffer (0.001\% Pluronic F68 in PBS). The two eyes of each mouse received different injections (vehicle, AAV2-CAG.SIRT1, AAV7m8-SNCG.SIRT1, AAV2-CAG. eGFP, or AAV7m8-ANCG.eGFP) allowing each eye to serve as an independent experimental end point.

\section{Optic nerve crush}

Optic nerve crush (ONC) was performed on 12-week-old C57Bl/6J wild-type mice as in our prior studies [4]. Mice were anesthetized systemically with xylazine and ketamine and topically with $0.5 \%$ proparacaine eye drops. Under a dissecting microscope, the conjunctiva was lifted with fine forceps and cut using scissors, exposing the sclera. Forceps were used to manipulate and retract orbital fat and muscles, allowing for exposure of the optic nerve. Injury to the optic nerve was induced using curved fine-tip forceps to induce a focal crush injury to the optic nerve $\sim 1$ to $2 \mathrm{~mm}$ behind the globe. Maximal pressure was used to close the forceps for $1 \mathrm{~s}$. Use of fine-tip forceps facilitated avoidance of orbital vessels to avoid ocular ischemia. Bleeding during the procedure was categorized as none, minimal, moderate, and large. Mice were excluded for more than moderate bleeding. In each experiment, the ONC was performed in one eye only, allowing the contralateral uninjured eye to serve as a control. The surgeon performing ONC was masked to eyes that received the AAV constructs.

\section{Optokinetic response recordings (OKRs)}

Visual function was assessed by measuring the OKR using commercial software and apparatus (OptoMetry; CerebralMechanics, Inc., Medicine Hat, AB, Canada) as previously described [15]. OKR was determined as the highest spatial frequency where mice track a $100 \%$ contrast grating that is projected at different spatial frequencies. Measurements were performed by an investigator masked to the experimental treatments. Each datapoint represents ten animals in experiments performed in triplicate.

\section{Retinal histology and RGC quantification}

Eyes were harvested and placed in 4\% PFA overnight at $48^{\circ} \mathrm{C}$. Eyes were washed in PBS followed by dissection of retinal cups. Tissues were permeabilized and blocked in $2 \%$ Triton X-100, 10\% normal bovine serum, and PBS and then incubated with goat anti-Brn3a antibody (Novus Biologics) diluted 1:100 at $48^{\circ} \mathrm{C}$. Retinal cups were washed and then incubated in secondary antibody solution containing $2 \%$ Triton X-100, 10\% normal bovine serum, and donkey antigoat AlexaFluor 594 antibody (1:500 dilution). After washing, samples were prepared as flat mounts and mounted onto glass slides with an aqueous mounting medium (SouthernBiotech) containing 4',6-diamidino-2-phenylindole (DAPI). RGCs were quantified as previously described $[5,6,16,17]$. Briefly, retinal micrographs were recorded at $\times 40$ magnification in 12 standard fields (from the center of the retina in each quadrant). Total RGC counts from the 12 fields per retinal sample covering a total area of $0.45 \mathrm{~mm}^{2} /$ retina were recorded by an investigator masked to the experimental conditions using Photometrics cell counting software. Retinal cross-sections were incubated in blocking buffer containing PBS, $2 \%$ Triton X-100, and 10\% normal donkey serum for $1 \mathrm{~h}$ at room temperature. Next, sections were incubated in primary antibody solution containing the previously described components and a rabbit anti-FLAG antibody (CST \#14793) at 1:100 dilutions overnight in a humidified chamber at room temperature. Sections were washed in PBS three times and incubated in secondary antibody solution containing donkey anti-rabbit AlexaFluor 488 antibody diluted at 1:200 for $2 \mathrm{~h}$ at room temperature. Slides were then washed in PBS three times and mounted with aqueous mounting medium (SouthernBiotech) containing DAPI. Retinal whole mount photography and counting of RGCs was performed by a masked investigator. Each experiment represents ten animals per group with experiments performed in triplicate.

\section{Axon analysis}

Neurofilament staining was performed and quantified in longitudinal paraffin embedded sections. Briefly, optic nerves were isolated, processed and embedded in paraffin. $5 \mu \mathrm{m}$ longitudinal paraffin sections were deparaffinized, rehydrated, and nonspecific binding was reduced using Blocking reagent (Vector Laboratories, Burlingame, CA, USA). Specimens were then incubated in rabbit antineurofilament antibody 1:500 (Abcam, Cambridge, MA, USA) at $4{ }^{\circ} \mathrm{C}$ overnight. Sections were washed three times with PBS, then incubated with anti-rabbit secondary antibody (Vectastain Elite ABC Rabbit kit) for $30 \mathrm{~min}$ at $37^{\circ} \mathrm{C}$. Avidin/Biotin Complex detection was performed by incubating with Vectastain Elite $\mathrm{ABC}$ reagent at $37^{\circ} \mathrm{C}$ for 30 min and DAB (diaminobenzidine, Vector labs) substrate for 3 min at RT followed by washing in running water for 5 min. Dehydrated slides were mounted using Refrax mounting medium. Photographs of three fields/nerve (one each at the distal, central, and proximal regions of the longitudinal optic nerve section) at $\times 40$ magnification were 
taken by a masked investigator. Neurofilament staining optical density was quantified by using ImageJ software (http://nih.gov).

Bisected optic nerves were incubated in $2 \%$ osmium tetroxide and dehydrated in graded ethanol immersions. The nerves were then embedded in epoxy resin Embed 812 (Electron Microscopy Sciences, Hatfield, PA), $0.75 \mu \mathrm{m}$ thick cross sections were generated from a section of the nerve $1.5 \mathrm{~mm}$ posterior to the globe and stained with $1 \%$ toluidine blue. Each optic nerve cross section was analyzed at five standardized photomicrographs $(75 \times 75 \mu \mathrm{m})$ obtained at $\times 100$ magnification: one in the center and in four quadrants. Axon counts were obtained by a masked operator using the AxonJ ImageAnalysis algorithm plugin for ImageJ (http://imagej.nih.gov/ij/plugins/axonj/) [18]. Mean \pm standard error of the mean (SEM).

\section{Statistics}

All data are represented as mean \pm SEM. Each experiment was repeated 3 times for statistical analysis. Differences between treatment groups with respect to OKR responses, RGC quantification, and optic nerve histopathology were compared using a 1-way ANOVA followed by Tukey's honest significant difference test using statistical software (GraphPad Prism 5.0; GraphPad Software, Inc., La Jolla, CA, USA). Differences were considered statistically significant at $P<0.05$. Data meet the assumption of normal distribution of tests with variances between GFP and SIRT1 groups. No randomization was performed; however investigators were masked to disease and therapeutic intervention to quantify endpoints.

\section{Results}

\section{Design and characterization of AAV7m8 vectors}

We generated AAV2 and AAV7m8 vectors expressing eGFP and a target gene human SIRT1 driven by the CAG promoter or a RGC-selective gamma synuclein promotor [13] (Fig. 1A1-4). Using induced pluripotent stem cell derived RGCs (IPS-RGCs) developed in a manner previously described [19] and collected from a healthy visually unaffected male, age 32, vector expression was examined in vitro using immunofluorescent labeling on iPSC-RGCs (Fig. 1B1-4). This revealed robust cell number (Fig B1) in cultures of viable iPS-RGCs (Fig. B2), with notable levels of gene expression with cytoplasmic and nuclear distribution of the tagged protein (Fig. B3).

Next, we compared the retinal transduction profile of AAV2-CAG.eGFP to AAV7m8-SNCG.eGFP following intravitreal delivery with a vector expressing enhanced green fluorescent protein in a cohort of wild-type mice as previously described [20]. This vector was compared to a previously described vector developed with a ubiquitous CAG promoter expressing the same human and codon optimized target gene SIRT1 but packaged in an AAV2 capsid. The AAV2.7m8-eGFP vector displayed a higher transduction efficiency of the ganglion cell layer than previously published [8]. Compared with the $25 \%$ RGC transduction displayed by the AAV2-CAG.eGFP vector, AAV7m8-SNCG.eGFP achieved $44 \%$ RGC transduction by quantifying the number of eGFP positive RGCs labeled with BRN3A antibody in retinal whole mounts (Fig. 2A1, A2). AAV7m8 vectors driving expression of SIRT1 were injected in to the right and left eyes, respectively, all wild-type mice displayed similar transduction profiles in vivo (Fig. B1 and B2).

\section{Constitutively expressed SIRT1 gene transfer using AAV2 vector does not rescue RGC protection after ONC}

C57B16/J mice received intravitreal injections of experimental or control AAV2 vectors at postnatal week 4 followed by optic nerve crush (ONC) induction at postnatal week 12 (Fig. 3A). Following ONC [4, 21] we measured visual function by OKR daily. Control treated animals treated post-intravitreal injections of AAV2-CAG.eGFP exhibit normal OKR scores (Fig. 3A) and retained RGCs (Fig. 3B, C) throughout the experimental timeline (Fig. 3A) which suggests no adverse effects associated with delivery or overexpression of the control transgene. Similarly, animals injected with AAV2-CAG.SIRT1 displayed strong responses prior to induction. Following optic nerve trauma, ONC animals exhibit a decline in OKR scores beginning at day 1 post trauma, which is sustained through the experimental timeline. AAV2-CAG.SIRT1 transfection demonstrated no significant protection of visual acuity throughout the experimental timeline (Fig. 3A).

Permanent visual decline observed in ONC uniformly results in loss of RGC numbers [4, 21]. Retinas from each treatment group were stained with antibodies directed against Brn3a to determine whether SIRT1 augmentation conferred a protective advantage upon RGCs during ONC (Fig. 3C). Intravitreal injection of AAV2 was well tolerated as indicated by comparative total RGC counts in shaminduced animals treated with eGFP. In mice traumatized with ONC, RGC numbers were significantly reduced in all treatment groups compared to sham-induced controls. This demonstrated no significant effect of SIRT1 overexpression using a ubiquitous CAG driven promoter on RGC survival that transfects only $24 \%$ of RGCs (Fig. 2). 
Fig. 1 Design and in vitro characterization of AAV2CAG and AAV7m8 SNCG vectors. A Outline of proviral expression cassettes used in the study. A1 and A2 illustrates comparative vector using AAV2 and $\mathrm{CAG}$ promoter with the cDNAs encoding eGFP or SIRT1 respectively. A3 and A4 Illustrates plasmid using the ganglion cell specific promoter, SNCG, driving cDNAs eGFP or SIRT1 respectively.

B Fluorescent micrographs of human SIRT1 protein expression in iPS-RGCs transduced with AAV7m8. SNCG.hSIRT1-3XFLAG. BlueDAPI nuclear stain, RedBRN3A nuclear stain, green3XFlag Tag epitope showing cytoplasmic and nuclear expression from the vector and blue/red/green merged images.
A1

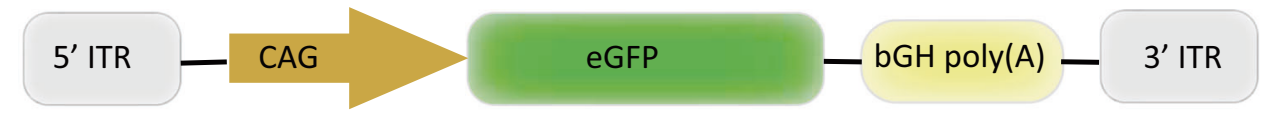

A2

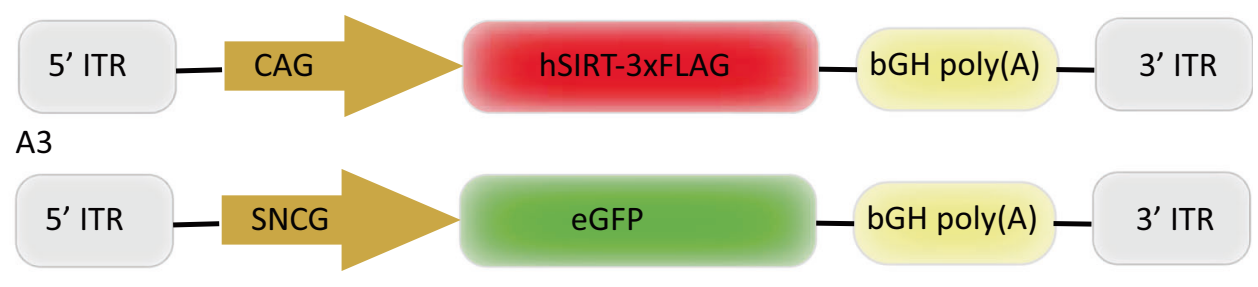

A4

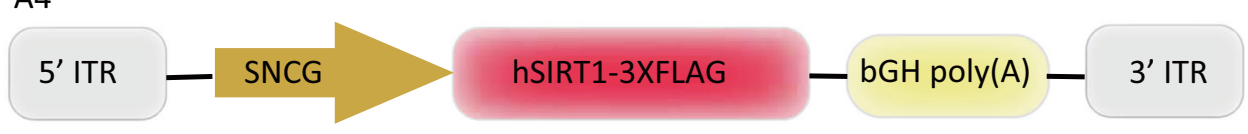

B
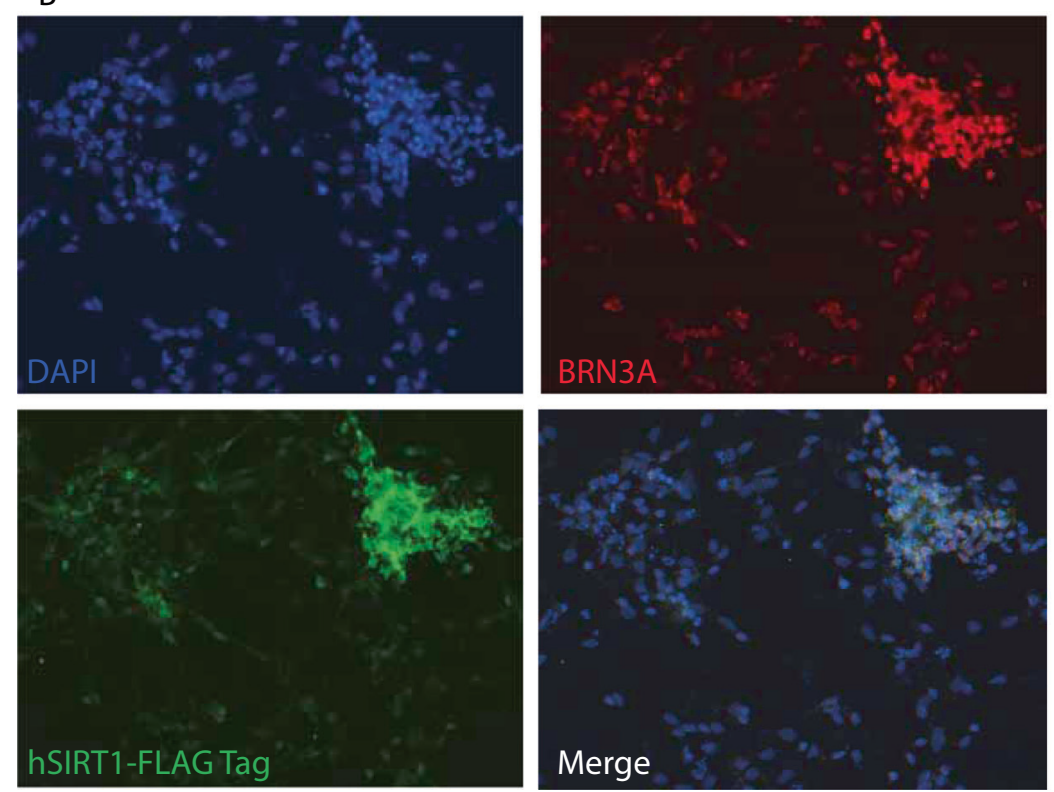

\section{Ganglion cell-selective expression of SIRT1 mediated by AAV7m8 vector with the SNCG delays loss of visual function and protects against RGC loss after ONC}

C57B16/J mice received intravitreal injections of AAV7m8 experimental and control vectors at postnatal week 4 followed by ONC at postnatal week 12. Sham Intravitreal injections of AAV2.7m8-eGFP did not interfere with OKR scores prior to ONC (Fig. 4A). Similarly, animals injected with AAV2-SNCG.SIRT1 displayed strong responses prior to induction. Following optic nerve trauma, untreated or AAV7m8-SNCG.eGFPtreated ONC animals exhibit a decline in OKR scores beginning at day 1 post trauma, which is sustained through the experimental timeline. However, eyes treated with AAV7m8-SIRT1 demonstrate a delay in reduction of functional responses by day 2 (Fig. 4A), (AAV2-CAG. SIRT $1=0.282 \pm 0.015 ; \quad$ AAV2-CAG.eGFP $=0.127 \pm$ $0.028 ; P=0.002)$. This effect was not sustained but was able to delay vision loss for 3 days.

Similar to experiments performed using AAV2-CAG mediated constitutive overexpression of SIRTI, retinas of each treatment group were stained with antibodies directed against Brn3a to determine whether SIRT1 augmentation conferred a protective advantage upon RGCs during ONC (Fig. 4B). Intravitreal injection of AAV7m8-SNCG was well tolerated as indicated by comparative total RGC counts in control AAV2.eGFP-injected animals. Treatment with AAV7m8-SNCG.SIRT1 resulted in a statistically significant increase in RGC survival compared to control eyes 
Fig. 2 AAV7m8 transduction profile and RGC transduction efficiency following intravitreal delivery.

A Representative micrograph of retinal flat mount following intravitreal injection of AAV7m8-SNCG.eGFP. RGCs are labeled with BRN3A (red). (inlet) Representative retinal flat mount used for calculating RGC transduction efficiency with AAV7m8. B Quantification of RGC transduction $(n=10$, experiments performed in triplicate; retinal whole mounts) comparing AAV2-CAG.SIRT1 vector. C Representative crosssection of mouse retina following intravitreal injection of AAV2-CAG.SIRT1. RGCs are labeled with Brn3a (red) and localized to the ganglion cell layer(GCL). Cells expressing the SIRT1 transgene are labeled green and also largely localized to the GCL.Data represented as mean \pm SEM.
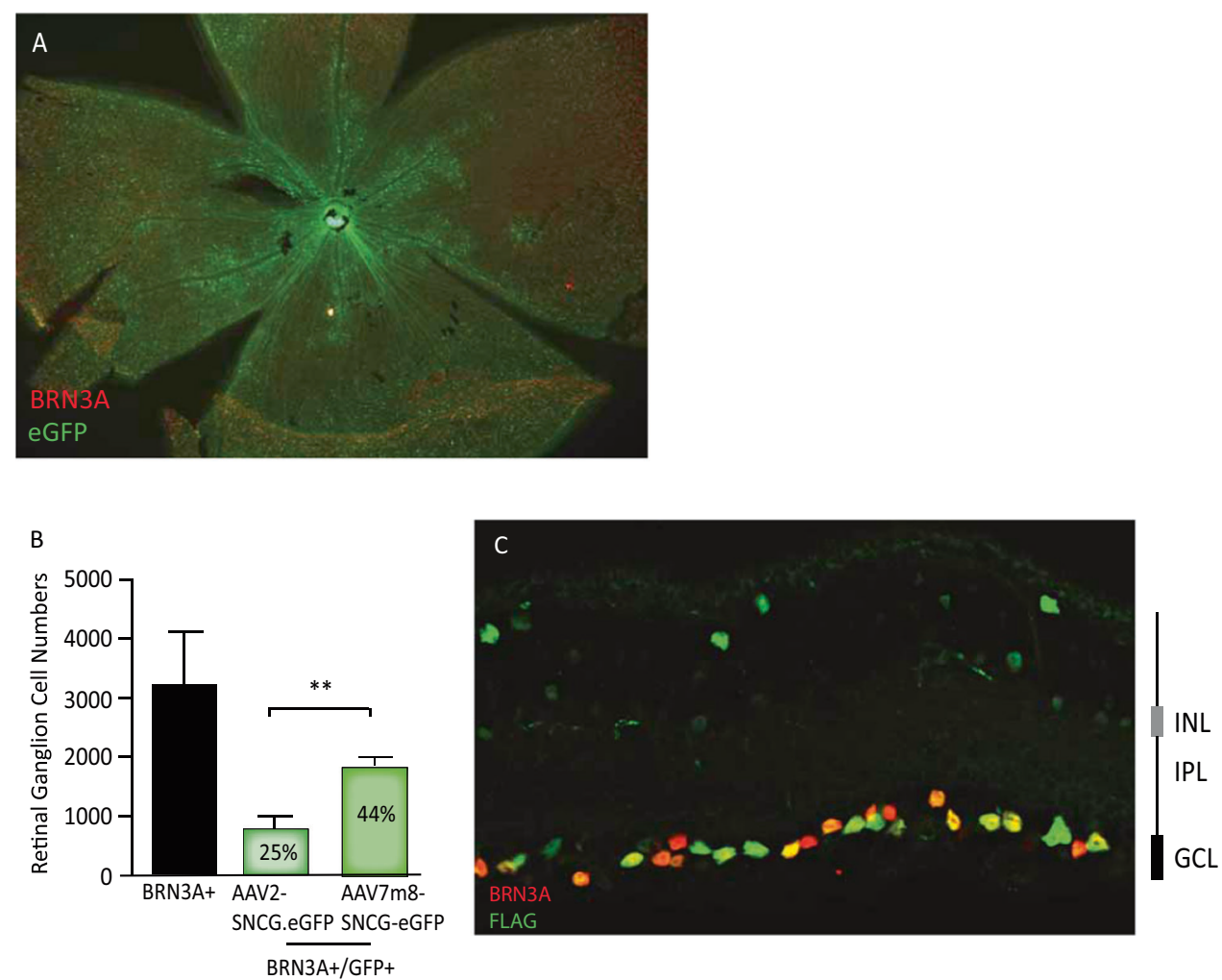

treated with AAV7m8-SNCG.eGFP (Fig. 4B, C) by day 6 after ONC (AAV7m8-SNCG.eGFP $=711.6 \pm 513.52$; AAV7m8-SNCG.SIRT1 $=1159.14 \pm 400.1 ; P=0.0156$ ).

\section{Discussion}

Adeno-associated virus (AAV) vectors have become the standard for achieving stable gene transfer with a safe clinical profile, especially when targeted to neurons, when taking into account numerous factors including dose, capsid, cassette, and manufacturing process. AAV2-vectors encoding RPE65 demonstrated a robust safety profile following subretinal delivery in human clinical trials for Leber congenital amaurosis type $2[22,23]$. Our study compared the neuroprotective effects of RGC specific to a non-cell specific gene transfer in an experimental mouse model of ONC. Our results suggest that SIRT1 driven by a RGC specific promoter delayed loss of visual acuity and enhanced RGC survival in ONC. Interestingly, even with notable loss of visual function (Fig. 3A) there are still significant RGCs present (Fig. 3B, C) suggesting that these cell bodies, although present, might be too damaged to convey visual signals. This finding begs further investigation into whether the constructs can facilitate regeneration of optic nerve axons over time. While using different transgene cassettes, both this change, as well as the cell selectivity resulted in a greater transduction efficiency as well as a more impressive therapeutic effect (Fig. 4). Results suggest that SIRT1 overexpression specifically in RGCs plays a significant role in delaying loss of RGC function and reducing RGC death from optic nerve injury. While we recognize that the AAV2 construct has a different transduction profile than AAV7m8 using the same transgene, the RGC layer, after intravitreal inject of the construct should receive the highest concentration of the virus. We previously showed that this AAV2 construct is transduced in multiple retinal layers [8] at low levels, but even with that current results with the AAV7m8 construct strongly suggest that it is the level expression of SIRT1 in RGCs that is is critical and sufficient to promote their survival. These effects are similar to neuroprotective effects promoted by pharmacologic and small molecular modulators of the SIRT1 pathway, as seen in mice treated with resveratrol and ST266 during experimental optic neuritis [21, 24]. Together, these results suggest that modulating SIRTI can promote RGC survival in multiple forms of optic neuropathy.

Under conditions of oxidative stress, SIRT1 is translocated to the nucleus and modulates activity of protein targets primarily involved in oxidative phosphorylation and mitochondrial biogenesis $[25,26]$. This function is induced by activating PGC1- $\alpha$, a transcription regulator of 


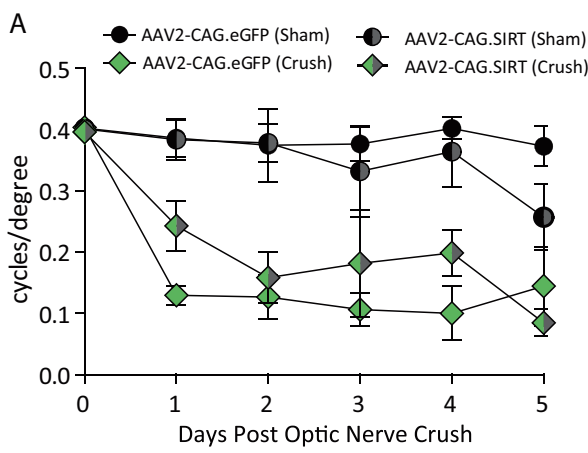

B

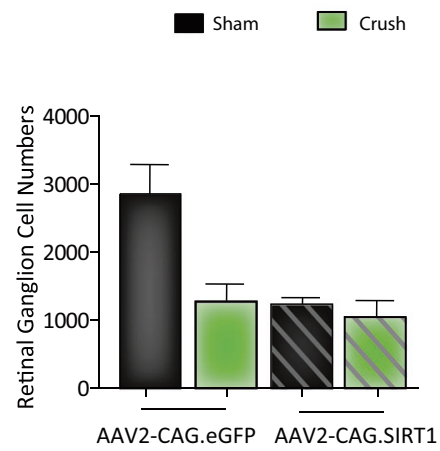

C

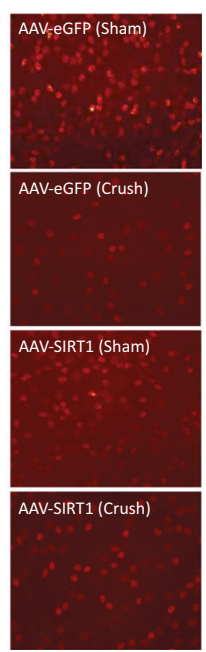

Fig. 3 Effect of AAV2 gene transfer on visual acuity and RGC in ONC. OKR recordings demonstrate significantly decreased visual acuity in eyes of ONC mice treated with AAV2-CAG.eGFP $(n=10$; experiments performed in tripicate). Treatment with AAV2- Mice treated with AAV2-CAG.SIRT1 $(n=12$; experiments performed in tripicate) show no significant effect on A visual acuity or B RGC survival by day 7. C Representative RGC counts by nuclear BRN3A staining. Data represented as mean \pm SEM. $* P<0.05$, $* * P<0.01$ by $1-$ way ANOVA with Tukey's HSD post-test.

mitochondrial function and antioxidant metabolism [27]. In addition to these known functions, active SIRT1 deacetylates and inhibits the transcription factor, p53, thereby downregulating apoptotic gene expression and thus improving cell viability [28]. Previous studies further demonstrate that preservation of RGCs through SIRT1 mechanisms involves reduction of oxidative stress. Studies with numerous mechanisms of cell stress to RGCs, including serum starvation, doxorubicin toxicity, and $\mathrm{H}_{2} \mathrm{O}_{2}$ exposure induced significant cell loss through increase of mitochondrial derived reactive oxygen species by proposed mechanisms involving promotion of mitochondrial biogenesis and function leading to increased superoxide dismutase activity [29]. With the combination of these mechanisms, SIRT1 could be important for promoting neuroprotection by activation of molecular pathways to inhibit cell damage from many different mechanisms of RGC damage.
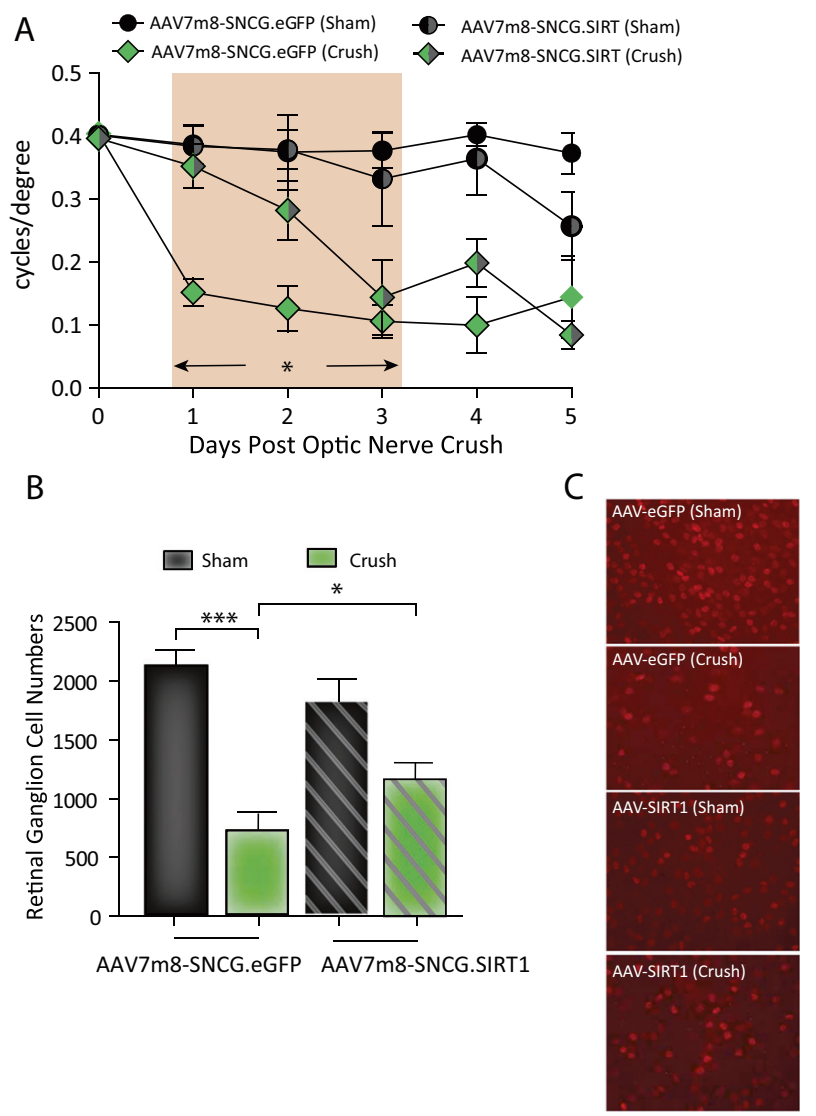

Fig. 4 Effect of AAV7m8 gene transfer on visual acuity and RGC in ONC. OKR recordings demonstrate significantly decreased visual acuity in eyes of ONC mice treated with AAV2-eGFP $(n=10$; experiments performed in triplicate) compared with Sham injured mice $(n=10$; experiments performed in tripicate $* * * p=0.002)$. A Treatment with AAV7m8-SIRT1 $(n=15$; experiments performed in tripicate) showed a significant delay in loss of visual function in ONC $(n=10$; experiments performed in tripicate) compared with Sham injured mice $(n=10$; experiments performed in tripicate) $(* p=0.03)$ on visual acuity seen in peach area outlined in the graph. B RGC flat mount counts demonstrate significantly decreased numbers in eyes of ONC mice treated with AAV2-eGFP $(n=10$; experiments performed in tripicate) compared with Sham injured mice $(n=10$; experiments performed in triplicate; $* * * p=0.001)$. Treatment with AAV7m8SIRT1 ( $n=10$; experiments performed in tripicate) showed a significant increase in retinal ganglion cell counted per flat month function in ONC $(n=7)$ compared with control ONC mice $(n=10$; experiments performed in tripicate) $(* p=0.03)$. C Representative RGC counts by nuclear BRN3A staining Data represented as mean \pm SEM. $* P<0.05, * * P<0.01$ by 1 -way ANOVA with Tukey's HSD post-test.

With ONC, we observed a statistically significant visual decline at day 1 post trauma with all AAV treated animals subjected to optic nerve insult, and this effect was significantly delayed in AAV7m8-SNCG.SIRT1 treated animals. Previously, Zuo et al. demonstrated a similar delay in loss of visual function after ONC in mice with constitutive overexpression of SIRT1 in all cell types using genetically modified mice. It was unclear from that prior study whether SIRT1 expression was necessary in multiple retinal cell 
types in order to promote RGC survival, as opposed to overexpression of SIRT1 mainly restricted RGCs. The similar effects on RGC survival found in the current study suggest a key role of SIRT1 signaling specifically within RGCs in SIRT1-mediated neuroprotection.

With a body of literature demonstrating that treatment with small molecular activators of the SIRT1 pathway, such as resveratrol, preserves visual function and RGC survival in mouse models of optic neuritis and optic nerve trauma [4-7, 17, 21, 24], the current results suggest that the AAV2.7m8-SIRT1 vector might have utility in multiple optic neuropathies. Future examination of potential neuroprotection in more chronic injury to the optic nerve, such as glaucoma, or acute injury, such as ischemic optic neuropathy, may be warranted. Our data demonstrate that with cell specificity using the SNGC promoter, protection of visual function and RGCs is better achieved, suggesting the importance of cell specificity in the design of potential neuroprotective gene therapies for optic neuropathies.

Acknowledgements The authors thank the CAROT research vector core for production of AAV vectors. Supported by National Institutes of Health Grants (EY019014, EY301163), RWJ-Harold Amos Faculty Development Award, Foundation Fighting Blindness, Brenda and Matthew Shapiro Stewardship, Robert and Susan Heidenberg Investigative Research Fund for Ocular Gene Therapy, Research to Prevent Blindness, Paul and Evanina Mackall Foundation Trust, Center for Advanced Retinal and Ocular Therapeutics, and the F. M. Kirby Foundation.

\section{Compliance with ethical standards}

Conflict of interest JB is a founder of Gensight Therapeutics and Limelight Bio and a scientific founder of Spark Therapeutics. JB receives a grant from Limelight Bio, is on the SAB for Akouos, and holds intellectual property relevant to this study. AGR, DSM, and KSS hold intellectual property relevant to this study. The other authors whose names are listed immediately below (RSK, TTD, KED, PA, VRMC) certify that they have NO affiliations with or involvement in any organization or entity with any financial interest (such as honoraria; educational grants; participation in speakers' bureaus; membership, employment, consultancies, stock ownership, or other equity interest; and expert testimony or patent-licensing arrangements), or non-financial interest (such as personal or professional relationships, affiliations, knowledge or beliefs) in the subject matter or materials discussed in this manuscript.

Publisher's note Springer Nature remains neutral with regard to jurisdictional claims in published maps and institutional affiliations.

Open Access This article is licensed under a Creative Commons Attribution 4.0 International License, which permits use, sharing, adaptation, distribution and reproduction in any medium or format, as long as you give appropriate credit to the original author(s) and the source, provide a link to the Creative Commons license, and indicate if changes were made. The images or other third party material in this article are included in the article's Creative Commons license, unless indicated otherwise in a credit line to the material. If material is not included in the article's Creative Commons license and your intended use is not permitted by statutory regulation or exceeds the permitted use, you will need to obtain permission directly from the copyright holder. To view a copy of this license, visit http://creativecommons. org/licenses/by/4.0/.

\section{References}

1. Jang SY. Traumatic optic neuropathy. Korean J Neurotrauma. 2018;14:1-5.

2. Kumaran AM, Sundar G, Chye LT. Traumatic optic neuropathy: a review. Craniomaxillofac Trauma Reconstr. 2015;8:31-41.

3. Tang Z, Zhang S, Lee C, Kumar A, Arjunan P, Li Y, et al. An optic nerve crush injury murine model to study retinal ganglion cell survival. J Vis Exp. 2011:2685. https://doi.org/10.3791/2685.

4. Zuo L, Khan RS, Lee V, Dine K, Wu W, Shindler KS. SIRT1 promotes RGC survival and delays loss of function following optic nerve crush. Invest Ophthalmol Vis Sci. 2013;54:5097-102.

5. Shindler KS, Ventura E, Dutt M, Elliott P, Fitzgerald DC, Rostami A. Oral resveratrol reduces neuronal damage in a model of multiple sclerosis. J Neuroophthalmol. 2010;30:328-39.

6. Shindler KS, Ventura E, Rex TS, Elliott P, Rostami A. SIRT1 activation confers neuroprotection in experimental optic neuritis. Invest Ophthalmol Vis Sci. 2007;48:3602-9.

7. Khan RS, Dine K, Wessel H, Brown L, Shindler KS. Effects of varying intranasal treatment regimens in ST266-mediated retinal ganglion cell neuroprotection. J Neuroophthalmol. 2019;39:191-9.

8. McDougald DS, Dine KE, Zezulin AU, Bennett J, Shindler KS. SIRT1 and NRF2 gene transfer mediate distinct neuroprotective effects upon retinal ganglion cell survival and function in experimental optic neuritis. Invest Ophthalmol Vis Sci. 2018;59:1212-20.

9. Chan L, Mahajan VB, Tsang SH. Genome surgery and gene therapy in retinal disorders. Yale J Biol Med. 2017;90:523-32.

10. DeBusk A, Moster ML. Gene therapy in optic nerve disease. Curr Opin Ophthalmol. 2018;29:234-8.

11. Yang W, Liu Y, Slovik KJ, Wu JC, Duncan SA, Rader DJ, et al. Generation of iPSCs as a pooled culture using magnetic activated cell sorting of newly reprogrammed cells. PLoS ONE. 2015;10: e0134995.

12. Teotia P, Chopra DA, Dravid SM, Van Hook MJ, Qiu F, Morrison $\mathrm{J}$, et al. Generation of functional human retinal ganglion cells with target specificity from pluripotent stem cells by chemically defined recapitulation of developmental mechanism. Stem Cells. 2017;35:572-85.

13. Chaffiol A, Caplette R, Jaillard C, Brazhnikova E, Desrosiers M, Dubus E, et al. A new promoter allows optogenetic vision restoration with enhanced sensitivity in Macaque retina. Mol Ther. 2017;25:2546-60.

14. Costello F, Coupland S, Hodge W, Lorello GR, Koroluk J, Pan YI, et al. Quantifying axonal loss after optic neuritis with optical coherence tomography. Ann Neurol. 2006;59:963-9.

15. Prusky GT, Alam NM, Beekman S, Douglas RM. Rapid quantification of adult and developing mouse spatial vision using a virtual optomotor system. Invest Ophthalmol Vis Sci. 2004;45:4611-6.

16. Quinn TA, Dutt M, Shindler KS. Optic neuritis and retinal ganglion cell loss in a chronic murine model of multiple sclerosis. Front Neurol. 2011;2:50.

17. Fonseca-Kelly Z, Nassrallah M, Uribe J, Khan RS, Dine K, Dutt $\mathrm{M}$, et al. Resveratrol neuroprotection in a chronic mouse model of multiple sclerosis. Front Neurol. 2012;3:84.

18. Zarei K, Scheetz TE, Christopher M, Miller K, Hedberg-Buenz A, Tandon A, et al. Automated axon counting in rodent optic nerve sections with AxonJ. Sci Rep. 2016;6:26559. 
19. Chavali VRM, Haider N, Rathi S, Alapati T, He J, Gill K, et al. Dual SMAD inhibition and Wnt inhibition enable efficient and reproducible differentiations of induced pluripotent stem cells into retinal ganglion cells. Sci Rep. 2020;10:11828.

20. Dudus L, Anand V, Acland GM, Chen SJ, Wilson JM, Fisher KJ, et al. Persistent transgene product in retina, optic nerve and brain after intraocular injection of rAAV. Vision Res. 1999;39:2545-53.

21. Grinblat GA, Khan RS, Dine K, Wessel H, Brown L, Shindler KS. RGC neuroprotection following optic nerve trauma mediated by intranasal delivery of amnion cell secretome. Investig Ophthalmol Vis Sci. 2018;59:2470-7.

22. Bennett J, Wellman J, Marshall KA, McCague S, Ashtari M, DiStefano-Pappas J, et al. Safety and durability of effect of contralateral-eye administration of AAV2 gene therapy in patients with childhood-onset blindness caused by RPE65 mutations: a follow-on phase 1 trial. Lancet. 2016;388:661-72.

23. Maguire AM, Simonelli F, Pierce EA, Pugh EN Jr., Mingozzi F, Bennicelli J, et al. Safety and efficacy of gene transfer for Leber's congenital amaurosis. N Engl J Med. 2008;358:2240-8.
24. Khan RS, Dine K, Bauman B, Lorentsen M, Lin L, Brown H, et al. Intranasal delivery of a novel amnion cell secretome prevents neuronal damage and preserves function in a mouse multiple sclerosis model. Sci Rep. 2017;7:41768.

25. Balaiya S, Abu-Amero KK, Kondkar AA, Chalam KV. Sirtuins expression and their role in retinal diseases. Oxid Med Cell Longev. 2017;2017:3187594.

26. Balaiya S, Ferguson LR, Chalam KV. Evaluation of sirtuin role in neuroprotection of retinal ganglion cells in hypoxia. Invest Ophthalmol Vis Sci. 2012;53:4315-22.

27. Nemoto S, Fergusson MM, Finkel T. SIRT1 functionally interacts with the metabolic regulator and transcriptional coactivator PGC-1 \{alpha\}. J Biol Chem. 2005;280:16456-60.

28. Luo J, Nikolaev AY, Imai S, Chen D, Su F, Shiloh A, et al. Negative control of p53 by Sir2alpha promotes cell survival under stress. Cell. 2001;107:137-48.

29. Khan RS, Fonseca-Kelly Z, Callinan C, Zuo L, Sachdeva MM, Shindler KS. SIRT1 activating compounds reduce oxidative stress and prevent cell death in neuronal cells. Front Cell Neurosci. 2012;6:63. 